\title{
Eficiência bioeconômica de vacas de diferentes níveis de produção de leite por lactação e estratégias de alimentação ${ }^{1}$
}

\author{
Francisco Palma Rennó ${ }^{2}$, José Carlos Pereira ${ }^{3}$, Carlos Antônio Moreira Leite ${ }^{4}$, Marcelo \\ Teixeira Rodrigues ${ }^{3}$, Oriel Fajardo de Campos $^{5}$, Dilermando Miranda da Fonseca ${ }^{3}$, Luciana \\ Navajas Rennó ${ }^{7}$
}

\footnotetext{
1 Pesquisa parcialmente financiada pelo CNPq.

2 Programa de Pós-graduação em Zootecnia - UFV, Viçosa, MG.

${ }^{3}$ Departamento de Zootecnia/UFV, Viçosa, MG. Bolsista do CNPQ.

${ }^{4}$ Departamento de Economia Rural/UFV, Viçosa, MG

${ }^{5}$ EMBRAPA Gado de Leite, Juiz de Fora, MG.

${ }^{6}$ Curso de Medicina Veterinária/UNIPAC, Juiz de Fora, MG.
}

RESUMO - Avaliou-se a eficiência bioeconômica de vacas de diferentes níveis de produção de leite por lactação submetidas a sete estratégias de alimentação. Utilizou-se um modelo de simulação desenvolvido com os programas CNCPS v5.0 e planilhas eletrônicas do Microsoft Excell ${ }^{\circledR}$, de forma a simular a produção e as exigências de nutrientes de uma lactação completa para vacas de diferentes níveis de produção. Foram realizadas avaliações da eficiência bioeconômica (EBE) em vacas de cinco níveis de produção de leite (10,15,20, 25 e $30 \mathrm{~kg} / \mathrm{dia})$, em sete estratégias de alimentação. A EBE foi definida de forma semelhante à eficiência biológica, considerando a quantidade de alimentos utilizada e a produção de leite obtida multiplicada pelos respectivos valores econômicos. Também com o propósito de avaliar a EBE das vacas de cada nível de produção, foram avaliados o produto físico médio (PFme) e o produto físico marginal (PFma) de cada nível de produção e de cada estratégia de alimentação. Na avaliação do PFma, foram estimadas equações de regressão da produção de leite por vaca considerando o nível de utilização de concentrados e os custos de alimentação. A eficiência bioeconômica de vacas de diferentes níveis de produção comprovou que a maior eficiência biológica de vacas de maior produção de leite por lactação não resultou, necessariamente, em maior eficiência bioeconômica. A eficiência de utilização de concentrados, quando avaliada em níveis crescentes de produção de leite por vaca, segue a lei dos retornos decrescentes e influencia a eficiência bioeconômica quanto maior o nível de produção de leite por vaca.

Palavras-chave: eficiência técnica e econômica, simulação, vacas leiteiras

\section{Bioeconomic efficiency of cows of different milk yield levels per lactation and feeding strategies}

\begin{abstract}
This work was carried out to evaluate the bioeconomic efficiency of cows of different levels of milk yield per lactation, under different feeding strategies. The bioeconomic evaluations was carried out in cows of five milk yield levels and seven feeding strategies The simulation model was developed with the programs CNCPS v5.0 and electronic spreadsheets of Microsoft Excell ${ }^{\circledR}$, in way to simulate the production and demands of nutrients of a complete lactation for cows of different milk yield levels. The bioeconomic efficiency (EBE) were evaluated in cows of five milk yield levels, in seven feeding strategies. The EBE was defined similarly to the biological efficiency, considering the amount of feeds utilized and the milk production obtained, multiplied by the respective economical values. Also, with the purpose of evaluating EBE of the cows of each production level, were evaluated the medium physical product (PFme) and the marginal physical product (PFma) for each production level and feeding strategy. In the evaluation of PFma, regression equations of milk yield per cow in function of concentrate level utilization and total feeding costs were estimate. The largest biological efficiency of cows of greater milk yield per lactation not result, necessarily, in larger bioeconomic efficiency. The efficiency of the use of concentrate, when evaluated in crescent milk yield per cows, follows the law of the decreasing returns and influences the bioeconomic efficiency as higher is the milk production level per cow.
\end{abstract}

Key Words: bioeconomic efficiency, dairy cows, simulation

Este artigo foi recebido em 1/2/2006 e aprovado em 8/10/2007.

Correspondências devem ser enviadas para francisco.renno@usp.br

* Endereço atual: Departamento de Nutrição e Produção Animal, Faculdade de Medicina Veterinária e Zootecnia da USP, Campus de Pirassununga, SP. 


\section{Introdução}

Eficiência biológica ou de produção é definida como a captura dos nutrientes dos alimentos, principalmente energia, para a produção de leite, reprodução e deposição de tecidos corporais e pode ser expressa como a porcentagem da energia bruta (EB) destinada a estes fins (Vandehaar, 1998). A eficiência de produção deve ser expressa em eficiência do uso da energia, nutriente mais limitante para vacas leiteiras modernas e cujo consumo está mais relacionado ao nível de produção de leite (Bauman et al., 1985).

Quanto mais uma vaca leiteira consome alimentos para suportar os aumentos crescentes da produção de leite, menos energia utilizará para satisfazer suas exigências de mantença, portanto, maior parte sobrará para síntese de leite. Esse fenômeno é denominado classicamente como "diluição das exigências de mantença” e ocorre com os aumentos do consumo de energia e da produção de leite, fatores comuns em vacas de maior mérito genético (Gibson, 1986).

Se vacas leiteiras apresentam produção individual de 12 a $15 \mathrm{~kg} / \mathrm{dia}$, grande proporção desses nutrientes é utilizada para a mantença desses animais e não para produção. Assim, uma vaca de $20 \mathrm{~kg} /$ dia, com mesmo peso vivo, é $40 \%$ mais eficiente que uma de $10 \mathrm{~kg} / \mathrm{dia}$ (NRC, 2001) e isto pode influenciar o custo final de produção, uma vez que a alimentação corresponde a aproximadamente $60 \%$ dos custos totais de produção (Ferreira, 2002). Além disso, quando a eficiência de utilização dos nutrientes é somada à demanda de energia para mantença em vacas mantidas em regime de pastagens, esses animais consomem 15 a $20 \%$ a mais de energia para a manutenção da atividade de pastejo (NRC, 2001), o que também pode aumentar os custos com alimentação.

Segundo Pereira (2000), diversos estudos em outros países demonstraram que vacas de maior potencial genético para produção de leite apresentam melhor retorno econômico, que pode variar de acordo com o sistema de produção utilizado. No entanto, apesar de a eficiência biológica ser influenciada pela maior utilização dos nutrientes para produção de leite em relação à utilização para mantença, uma vaca de maior produção não é necessariamente mais eficiente economicamente que outra de menor produção, considerando a relação de preços de leite e de insumos no Brasil.

De acordo com Vandehaar (1998), a avaliação das relações entre o nível de produção e o nível de rentabilidade deve incluir dois fatores principais. O primeiro refere-se aos custos de alimentação, que podem ser maiores dependendo do nível de produção; e o segundo é a diluição dos custos fixos relativos à produção total de leite quando a produção por vaca é aumentada.

Pereira (2000) cita que o retorno financeiro, em relação ao custo de alimentação, constitui o maior determinante dos lucros ou das perdas na exploração leiteira. Esse fator pode variar de acordo com os preços de venda do leite, com o custo de alimentação e com a resposta da vaca ao alimento fornecido. Segundo esse autor, a obtenção de maior retorno econômico depende do custo dos nutrientes nos alimentos disponíveis e do valor da venda do leite.

Assim, resultados positivos do aumento do nível de produção de leite influenciando a eficiência biológica de vacas leiteiras podem ser diferentes quando avaliada a eficiência bioeconômica. Na eficiência bioeconômica, são considerados os custos e as receitas decorrentes da alimentação e da produção de leite, respectivamente. Como a receita é influenciada pela relação de preços de insumos e de produtos, o aumento do nível de produção por vaca pode não resultar em aumentos de eficiência bioeconômica.

No Brasil são poucos os estudos para avaliar o efeito do aumento da eficiência biológica de vacas leiteiras, ou aumento médio da produção de leite por animal, sobre o retorno econômico com a produção de leite. Dessa forma, avaliou-se neste estudo a eficiência bioeconômica de vacas de diferentes níveis de produção de leite por lactação (eficiência biológica) submetidas a sete estratégias de alimentação.

\section{Material e Métodos}

Uma plataforma computacional com a produção de leite de uma lactação completa, desenvolvida utilizando-se o software CNCPS (Cornell Net Carbohydrate and Protein System), versão 5.0 (Fox et al., 2003), foi utilizada para formulação das dietas. Com os resultados, por meio de planilhas eletrônicas e utilizando-se o software Microsoft EXCELL ${ }^{\circledR}$, foram realizados os cálculos de eficiência bioeconômica de vacas de diferentes níveis de produção de leite submetidas a sete estratégias de alimentação.

A plataforma computacional utilizada considera as necessidades nutricionais de vacas leiteiras durante o período de uma lactação, combinando o valor nutritivo e as características agronômicas das forrageiras utilizadas, para vacas de cinco níveis de produção. Modificações nas necessidades dietéticas das vacas decorrentes do aumento de produção ou da modificação da base forrageira resultam em alterações no nível de utilização de forragens e de 
concentrado alterando a produtividade e a eficiência bioeconômica. A descrição detalhada do modelo de simulação utilizado foi descrita por Rennó et al. (2008).

Considerou-se que o animal era da raça Holandesa, tinha idade adulta de 48 meses, peso vivo médio de $600 \mathrm{~kg}$ após o parto e que ficaram prenhes com 120 dias pós-parto, portanto, com intervalo de partos de 13,2 meses. Foram considerados oito períodos de lactação para obtenção da produção de leite e das exigências nutricionais e para a formulação das dietas: 14, 42, 70, 112, 168, 231, 294 e 305 dias de lactação.

Para estimativa da produção média de leite em cada período de lactação, adotou-se o modelo proposto por Congleton \& Everett (1980). Avaliaram-se animais de cinco níveis de produção de leite por lactação: 3.075,6; 4.473,7; 6.151,3; 7.549,3; e 9.227,0 kg de leite, denominados, respectivamente, nível de produção baixo (B), médio baixo (MB), médio (M), médio-alto (MA) e alto (A), correspondentes a produções médias de 10,1; 14,7; 20,2; 24,8 e 30,3kg/dia em 305 dias de lactação. Considerou-se que as produções de leite se referem à composição de 3,8\% de gordura e 3,3\% de proteína total.

No cálculo da produção total de leite na lactação, foram utilizadas as médias das produções de leite, estimadas segundo o modelo citado anteriormente, e calculada a média entre dois pontos consecutivos multiplicada pelo número de dias do período para obtenção das produções parciais ao longo da lactação. A produção final correspondeu ao somatório dessas produções parciais.

Foi utilizado o software CNCPS, versão 5.0 (Fox et al., 2003), no cálculo das dietas e na estimativa das exigências nutricionais e do valor nutritivo dos alimentos. $\mathrm{Na}$ estimativa do valor nutritivo dos alimentos, foram utilizadas as equações do Nível 1. Exigiu-se que todas as dietas formuladas atendessem às recomendações de balanço de nutrientes para a fase da lactação, independentemente do volumoso ou dos componentes dos concentrados utilizados.

Para avaliação dos volumosos, foram desenvolvidas sete estratégias de alimentação para uma lactação completa, variando entre sistemas de alimentação com um único volumoso ao longo do ano a sistemas de alimentação nos quais são utilizadas pastagens na época das águas (outubro a março) como volumoso exclusivo e suplementação com silagem ou cana-de-açúcar na época das secas (abril a setembro): estratégia (EST) 1 - silagem de milho (SIM) como volumoso exclusivo, ao longo de toda lactação (SIM); EST 2 - SIM durante a época seca e pastejo em capim-braquiária (BRI) durante a época das águas (SIM+BRI); EST 3 - SIM durante a época seca e pastejo de capim-elefante (NAP) durante a época das águas (SIM+NAP); EST 4 - SIM durante a época seca e pastejo em capim-tifton-85 (TIF) durante a época das águas (SIM+TIF); EST 5 - cana-deaçúcar (CAN) durante a época seca e pastejo de BRI durante a época das águas (CAN+BRI); EST 6 - CAN durante a época seca e pastejo de NAP durante a época das águas (CAN+NAP); EST 7 - CAN durante a época seca e pastejo de TIF durante a época das águas (CAN+TIF).

Os preços do leite e dos insumos foram obtidos por meio da Scott Consultoria (2003) e corresponderam ao período de janeiro a dezembro de 2003. Nas análises econômicas, foram utilizados os preços deflacionados até dezembro de 2003 e os preços mensais para determinação dos custos de alimentação e venda de leite, de acordo com a variação mensal de preços.

No cálculo da quantidade de alimentos fornecida e dos custos com alimentação ao longo da lactação, foram consideradas as médias das dietas formuladas entre dois pontos na curva de lactação, segundo metodologia descrita por Rennó et al. (2008). Os custos de alimentação foram obtidos considerando as exigências de alimentos para cada período de lactação avaliado e os preços dos insumos nesse período, de acordo com a formulação de ração. O gasto diário em determinada fase da lactação foi multiplicado pelo número de dias da fase, e assim sucessivamente, para a composição dos custos totais de alimentação ao final da lactação.

Todas as dietas foram formuladas para o completo atendimento das exigências nutricionais de mantença, produção, gestação e mobilização/reposição de reservas corporais, considerando uma lactação completa. Assim, foram consideradas não somente as exigências de energia, como também as de proteína e de fibra. Em cada nível de eficiência biológica (ou produção de leite) com a utilização das dietas simuladas, foi avaliada a eficiência bioeconômica (EBE) das vacas de diferentes níveis de produção de leite em cada estratégia de alimentação.

A EBE foi definida de forma semelhante à eficiência biológica, proposta por Vandehaar (1998), considerando ainda a quantidade de alimento utilizada e a produção de leite obtida, multiplicada pelos respectivos valores econômicos. Dessa forma, a EBE foi calculada considerando os custos de alimentação e a renda bruta com a venda de leite utilizando-se a equação:

$$
E B E=100-\frac{\left(C A_{i j} * 100\right)}{R B P L_{j}}
$$

em que CA é o custo de alimentação ( $R$ \$) da estratégia i no nível de produção j; e RBPL é a renda bruta (R\$) da venda 
de leite no nível de produção j. Quanto maior o valor de EBE, maior o percentual de receita e menores os custos com alimentação, portanto, um resultado favorável.

Também com o propósito de avaliar a eficiência bioeconômica das vacas de cada nível de produção, avaliou-se o produto físico médio (PFme) ou a produtividade média de cada nível de produção em cada estratégia de alimentação. Utilizando-se o PFme, é possível avaliar a eficiência do processo de produção, ou seja, a quantidade da produção (leite) dividida pela quantidade de insumo (concentrado) utilizado, conforme equação a seguir:

$$
\text { PFme }_{X_{1}}=\frac{Y}{X_{1}}
$$

em que Y é a quantidade de produto (produção de leite); e $\mathrm{X}_{1}$ a quantidade do insumo utilizado (concentrado).

Também foi avaliada a eficiência técnica de produção, pela relação entre a produção de leite obtida e o custo dos concentrados (L/R \$conc), e pela produção de leite em relação aos gastos com alimentação (L/R \$alim).

Avaliou-se o produto físico marginal (PFma) ou a produtividade marginal de cada estratégia de alimentação. O PFma refere-se à quantidade que cada unidade adicional do fator variável acrescenta ao produto físico total. Dessa forma, é possível avaliar a resposta na produção de leite aos custos de alimentação para cada estratégia de alimentação considerando todos os níveis de produção avaliado, conforme equação a seguir:

$$
P F m a=\frac{\partial Y}{\partial X_{1}}
$$

em que Y é a derivada parcial da mudança na produção de leite e $\mathrm{X}_{1}$ é a derivada parcial do acréscimo em uma unidade adicional do fator variável (kg concentrado, R\$ gasto com concentrados e R\$ gasto com alimentação).

Para a avaliação do PFma, foram estimadas equações de regressão da produção de leite por vaca em relação ao nível de concentrados, avaliado em kg/vaca/ano e R \$/vaca/ano, e dos custos de alimentação (R\$/vaca/ano). Na estimativa das equações de regressão, utilizou-se o software SAS com o procedimento PROC REG (SAS, 1999).

\section{Resultados e Discussão}

A eficiência bioeconômica (EBE) variou de 31,46 a 51,83\% (Tabela 1), considerando vacas de baixo e alto nível de produção. Na média, não foram observadas modificações significativas na EBE quando o nível de produção de leite por vaca aumentou; os valores foram de 43,80; 48,72; 47,23; 48,59 e 51,28\%, respectivamente, nos níveis baixo, médio-baixo, médio, médio-alto e alto (nível alto somente para a estratégia SIM).

O padrão de resposta da EBE ao aumento no nível de produção diferiu entre as estratégias de alimentação. Na estratégia com alimentação à base de silagem de milho como volumoso exclusivo (SIM), a EBE foi maior, em virtude do aumento do nível de produção, que ocasionou diferença de 63,88\% na eficiência entre os níveis de produção baixo e médio-alto. Entretanto, nas estratégias com alimentação à base de silagem de milho e pastagens (SIM+BRI, SIM+NAP e SIM+TIF), foi observado aumento de aproximadamente $10 \%$ quando o nível de produção foi de baixo para médiobaixo. A partir desse nível de produção, foram observadas pequenas mudanças na EBE, de 0,56 a 4,50\%, entre os níveis de produção médio-baixo, médio e médio-alto.

Nas estratégias com alimentação à base de cana-deaçúcar e pastagens (CAN+BRI, CAN+NAP e CAN+TIF), foi observado aumento médio de $6,54 \%$ na EBE quando avaliados os níveis baixo e médio-baixo. A partir do nível médiobaixo de produção, não foram observadas mudanças significativas na EBE quando aumentado o nível de produção de leite por vaca. Os níveis médio e médio-alto apresentaram EBE de 99,64 e 100,16\% em relação ao nível baixo de produção (Tabela 1).

Apesar da maior eficiência biológica de vacas de maior produção de leite por lactação, o que resultou em maior direcionamento proporcional dos nutrientes para a produção de leite em relação à mantença (NRC, 2001; Fox et al., 2003), neste estudo, não foram verificados benefícios do aumento de produção na eficiência bioeconômica na mesma proporção observada na eficiência biológica. Esses resultados podem ser atribuídos à relação de preços de insumos utilizados na alimentação, em virtude do preço de leite praticado no mesmo período avaliado. Assim, os benefícios de se utilizar vacas de maior potencial de produção de leite poderiam estar relacionados a outros fatores, como maior escala de produção e diluição dos custos fixos dos sistemas de produção de leite, e não diretamente à maior eficiência biológica desses animais.

A utilização do concentrado na avaliação do produto físico médio (PFme) e da eficiência técnica ( $\mathrm{L} / \mathrm{R}$ \$conc) seguiu a lei dos retornos decrescentes ou da produtividade decrescente (Tabela 2). Verificou-se produtividade decrescente do fator variável (os concentrados) quando, a cada nova unidade do investimento ( $\mathrm{kg}$ de concentrado), o aumento na produção de leite foi inferior ao da unidade anterior (Figura 1a, b).

Contudo, quando avaliada a eficiência técnica da produção de leite em relação ao gasto com alimentação (L/R\$alim), houve efeito da estratégia de alimentação sobre 
Tabela 1 - Eficiência bioeconômica de vacas de diferentes níveis de produção de leite por lactação (305 dias) em cada estratégia de alimentação

\begin{tabular}{lccccc}
\hline \multirow{2}{*}{$\begin{array}{l}\text { Estratégia de } \\
\text { alimentação }\end{array}$} & \multicolumn{5}{c}{ Nível de produção } \\
\cline { 2 - 6 } & Baixo $^{1}$ & Médio-baixo & Médio & Médio-Alto & Alto \\
\hline \multicolumn{5}{c}{ Custo de } & alimentação/Renda bruta \\
SIM & 31,46 & 42,80 & 48,18 & 51,55 & 51,28 \\
SIM+BRI & 44,14 & 48,91 & 48,20 & 50,46 & \\
SIM+NAP & 48,26 & 52,12 & 49,98 & 51,83 & \\
SIM+TIF & 47,28 & 52,94 & 49,41 & 50,78 & \\
CAN+BRI & 42,89 & 46,13 & 44,63 & 45,19 & \\
CAN+NAP & 47,28 & 48,97 & 45,85 & 46,24 & \\
CAN+TIF & 45,31 & 49,15 & 44,36 & 44,09 & \\
\hline & \multicolumn{5}{c}{ Custo de alimentação/Renda bruta ${ }^{1}$} \\
SIM & 100,00 & 136,07 & 153,16 & 163,88 & 163,03 \\
SIM+BRI & 100,00 & 110,81 & 109,19 & 114,33 & \\
SIM+NAP & 100,00 & 107,99 & 103,56 & 107,39 & \\
SIM+TIF & 100,00 & 111,97 & 104,51 & 107,40 & \\
CAN+BRI & 100,00 & 107,56 & 104,06 & 105,36 & \\
CAN+NAP & 100,00 & 103,58 & 96,96 & 97,80 & \\
CAN+TIF & 100,00 & 108,48 & 97,90 & 97,32 &
\end{tabular}

${ }^{1}$ Níveis baixo, médio-baixo, médio, médio-alto e alto correspondem a médias de produção de leite por lactação (305 dias) de 10, 15, 20, 25 e $30 \mathrm{~kg} / \mathrm{dia}$, respectivamente.

2 Relativização da eficiência bioeconômica em relação ao nível baixo de produção de leite por vaca (linha).

$\mathrm{SIM}=$ silagem de milho como volumoso exclusivo; $\mathrm{SIM}+\mathrm{BRI}=$ silagem de milho durante a época seca e pastejo em capim-braquiária durante a época das águas; SIM+NAP = silagem de milho durante a época seca e pastejo de capim-elefante durante a época das águas; SIM+TIF = silagem de milho durante a época seca e pastejo em capim-tifton 85 durante a época das águas; $\mathrm{CAN+BRI} \mathrm{=} \mathrm{cana-de-açúcar} \mathrm{durante} \mathrm{a} \mathrm{época} \mathrm{seca} \mathrm{e} \mathrm{pastejo} \mathrm{em}$ capim-braquiária durante a época das águas; $\mathrm{CAN}+\mathrm{NAP}=$ cana-de-açúcar durante a época seca e pastejo de capim-elefante durante a época das águas; CAN+TIF = cana-de-açúcar durante a época seca e pastejo em capim-tifton 85 durante a época das águas.

a produtividade do fator variável. A avaliação da L/R \$alim revelou resultados semelhantes à avaliação da EBE descrita anteriormente. Na estratégia SIM, houve retorno crescente quando avaliada a eficiência técnica em relação aos custos de alimentação (Tabela 2).

Nas estratégias com alimentação à base de silagem de milho e pastagens (SIM+BRI, SIM+NAP e SIM+TIF), houve aumento da eficiência técnica somente quando comparado o nível médio-baixo ao nível baixo de produção. Os níveis médio e médio-alto apresentaram resultados semelhantes ao médio-baixo, uma vez que a eficiência técnica desses níveis de produção não aumentou. Nas estratégias à base de canade-açúcar, o comportamento foi semelhante entre os níveis de produção avaliados, comprovando retornos constantes ou aumento na produtividade constante quando o nível de produção de leite por vaca foi maior (Figura 2).

Segundo o NRC (2001) e Fox et al. (2003), entre as fontes de variação da eficiência biológica de vacas leiteiras, a partição dos nutrientes dietéticos em leite apresenta a maior importância. A relação entre a exigência nutricional de
Tabela 2 - Produto físico médio da produção de leite por animal em função do nível de utilização de concentrado $(\mathrm{kg} /$ vaca/ano) e eficiência técnica da produção de leite em função do gasto com concentrados (L/R \$conc) e alimentação (L/R\$alim), em $R \$ / v a c a / a n o$, segundo o nível de produção em cada estratégia de alimentação

\begin{tabular}{|c|c|c|c|c|c|}
\hline \multirow{2}{*}{$\begin{array}{l}\text { Estratégia de } \\
\text { alimentação }\end{array}$} & \multicolumn{5}{|c|}{ Nível de produção } \\
\hline & Baixo $^{1}$ & Médio-baixo & Médio & Médio-Alto & Alto \\
\hline & \multicolumn{5}{|c|}{ Concentrado (kg/vaca/ano) } \\
\hline SIM & 3,68 & 4,10 & 3,39 & 3,43 & 3,04 \\
\hline SIM+BRI & 4,21 & 3,35 & 2,68 & 2,69 & \\
\hline $\mathrm{SIM}+\mathrm{NAP}$ & 4,25 & 3,21 & 2,62 & 2,64 & \\
\hline SIM+TIF & 4,34 & 3,47 & 2,68 & 2,62 & \\
\hline $\mathrm{CAN}+\mathrm{BRI}$ & 3,03 & 2,74 & 2,38 & 2,36 & \\
\hline $\mathrm{CAN}+\mathrm{NAP}$ & 3,25 & 2,64 & 2,29 & 2,34 & \\
\hline \multirow[t]{2}{*}{ CAN+TIF } & 3,00 & 2,73 & 2,34 & 2,32 & \\
\hline & \multicolumn{5}{|c|}{$\mathrm{L} / \mathrm{R} \$$ conc } \\
\hline SIM & 5,84 & 6,65 & 6,23 & 6,27 & 5,57 \\
\hline SIM+BRI & 6,51 & 5,82 & 5,12 & 5,13 & \\
\hline SIM+NAP & 7,91 & 6,44 & 5,38 & 5,32 & \\
\hline SIM+TIF & 8,49 & 6,85 & 5,45 & 5,27 & \\
\hline $\mathrm{CAN}+\mathrm{BRI}$ & 4,99 & 4,55 & 4,22 & 4,15 & \\
\hline $\mathrm{CAN}+\mathrm{NAP}$ & 5,85 & 4,92 & 4,36 & 4,27 & \\
\hline \multirow[t]{2}{*}{ CAN+TIF } & 5,85 & 5,07 & 4,33 & 4,14 & \\
\hline & \multicolumn{5}{|c|}{ L/R\$alim } \\
\hline SIM & 3,01 & 3,61 & 3,99 & 4,26 & 4,24 \\
\hline SIM+BRI & 3,70 & 4,04 & 3,99 & 4,17 & \\
\hline $\mathrm{SIM}+\mathrm{NAP}$ & 3,99 & 4,31 & 4,13 & 4,29 & \\
\hline SIM+TIF & 3,92 & 4,39 & 4,08 & 4,20 & \\
\hline CAN+BRI & 3,62 & 3,83 & 3,73 & 3,77 & \\
\hline $\mathrm{CAN}+\mathrm{NAP}$ & 3,92 & 4,05 & 3,81 & 3,84 & \\
\hline CAN+TIF & 3,78 & 4,06 & 3,71 & 3,69 & \\
\hline
\end{tabular}

1 Níveis baixo, médio-baixo, médio, médio-alto e alto correspondem a médias de produção de leite por lactação (305 dias) de 10, 15, 20, 25 e $30 \mathrm{~kg} / \mathrm{dia}$, respectivamente.

$\mathrm{SIM}=$ silagem de milho como volumoso exclusivo; $\mathrm{SIM}+\mathrm{BRI}=$ silagem de milho durante a época seca e pastejo em capim-braquiária durante a época das águas; SIM+NAP = silagem de milho durante a época seca e pastejo de capim-elefante durante a época das águas; SIM+TIF = silagem de milho durante a época seca e pastejo em capim-tifton 85 durante a época das águas; $\mathrm{CAN}+\mathrm{BRI}$ = cana-de-açúcar durante a época seca e pastejo em capim-braquiária durante a época das águas; CAN+NAP = cana-de-açúcar durante a época seca e pastejo de capim-elefante durante a época das águas; CAN+TIF = cana-de-açúcar durante a época seca e pastejo em capim-tifton 85 durante a época das águas.

mantença e a utilizada para a produção de leite define a eficiência biológica de vacas leiteiras e, dessa forma, vacas de mesmo peso corporal (mesma exigência de mantença)e com maior produção de leite apresentam maior eficiência de utilização de nutrientes dos alimentos e, conseqüentemente, melhor eficiência biológica que vacas de maior produção (Vandehaar, 1998).

No entanto, apesar da maior eficiência biológica das vacas de maior produção, a eficiência bioeconômica é influenciada por outros fatores além do nível de produção de leite. São considerados as quantidades e os custos dos nutrientes utilizados, assim como a receita com o produto obtido, dentro de um cenário específico de produção. 
Assim, a relação de troca entre os diversos insumos utilizados e a produção de leite, bem como as necessidades dietéticas em cada nível de produção, influencia de forma mais contundente o resultado que a maior eficiência biológica de vacas de maior produção de leite (Tabelas 1 e 2).

Nessa avaliação, as vacas de maior produção e, conseqüentemente, de maior eficiência biológica, não apresentaram melhor eficiência bioeconômica, mesmo quando consideradas vacas de produções de leite por lactação diferentes $(10,15,20,25$ e 30 kg/dia) (Tabela 1). Com exceção da comparação entre os níveis baixo e médio-baixo, os demais níveis de produção apresentaram EBE semelhantes.

Uma possível explicação para esses resultados é a gradativa diminuição do PFme e da eficiência técnica quando avaliada a utilização dos concentrados em cada nível de produção. São obtidos retornos decrescentes na utilização dos concentrados (kg/vaca/ano) com o aumento do nível de produção (Figura 1a, b). Como o concentrado representa

A

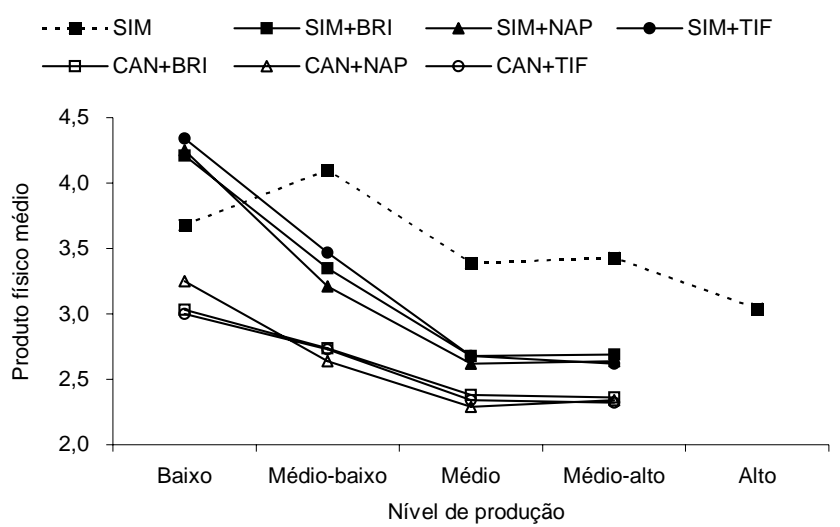

B

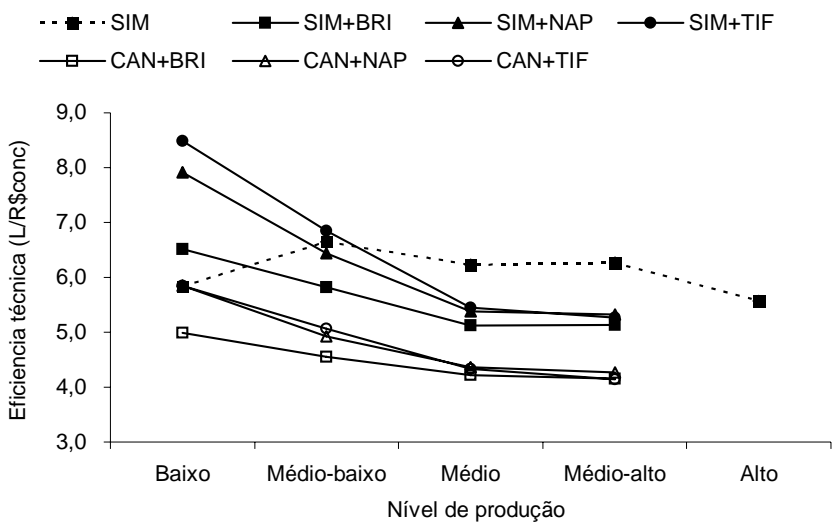

Figura 1 - Produto físico médio e eficiência técnica da produção de leite (PL/vaca/ano), em cada nível de utilização de concentrado (em kg/vaca/ano e $\mathrm{R} \$ / \mathrm{vaca} / \mathrm{ano}$, respectivamente), segundo o nível de produção, para cada estratégia de alimentação. significativa parcela dos custos de alimentação de vacas em lactação, sendo ainda maior com o aumento do nível de produção (Rennó et al., 2008), as reduções na eficiência de utilização dos concentrados comprometem o melhor resultado na EBE de vacas de maior produção na relação de preços de insumos e do leite utilizada neste estudo.

Pereira (2000) citou que ocorre redução da produtividade com a utilização de concentrados para vacas de maiores produções de leite e que os acréscimos na utilização de concentrado não correspondem à resposta crescente na produção de leite na mesma proporção. Dessa forma, quando o nível de produção de leite aumenta, ocorre perda na eficiência de utilização dos concentrados a cada litro de leite produzido para todas as estratégias de alimentação avaliadas.

Bath \& Sosnik (1992) relataram que a formulação de dietas para maximização da receita sobre os custos de alimentação deve incluir dois fatores principais: o preço do leite e o princípio dos retornos decrescentes. Esses autores relataram que a produção de leite por unidade de nutriente dietético é influenciada pelo nível de produção. Quanto maior a produção de leite por vaca, menor a resposta produtiva por unidade de insumo utilizado. Além disso, na análise realizada neste estudo, não foi considerado o efeito da redução dos custos fixos, por vaca, com o aumento da produção por lactação. Assim, esses resultados se referem à avaliação dos sistemas de alimentação e não incluem todo o sistema de produção.

Todas as equações da produção de leite por animal (kg/ vaca/ano), em cada nível de concentrado, e dos custos de alimentação utilizadas para calcular o produto físico marginal (PFma) de cada estratégia de alimentação apresentaram alto

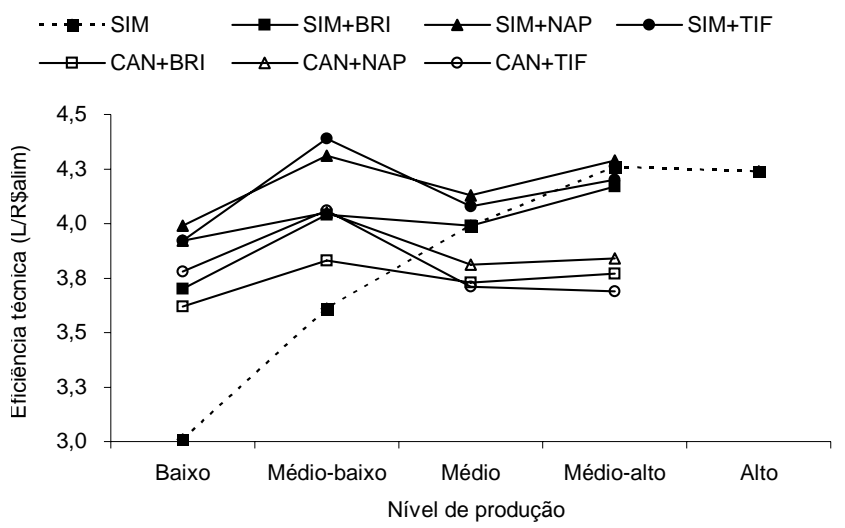

Figura 2 - Eficiência técnica da produção de leite (PL/vaca/ano) em relação ao custo de alimentação (R\$/vaca/ano) segundo o nível de produção, em cada estratégia de alimentação. 
coeficiente de determinação (Tabela 3), comprovando bom ajuste aos dados avaliados.

O PFma, que avaliou a utilização de concentrados, demonstrou maior resposta no uso deste insumo, nas duas formas avaliadas (kg/vaca/ano e R \$/vaca/ano), para a estratégia de alimentação com a silagem de milho como volumoso exclusivo (SIM). Com a utilização da estratégia SIM, espera-se na média de todos os níveis de produção uma produção de leite de 3,06 e 6,35 L/kg de concentrado utilizado ou por R\$ gasto com concentrado, respectivamente.

As estratégias com alimentação à base de silagem de milho e pastagens (SIM+BRI, SIM+NAP e SIM+TIF) ou cana-de-açúcar e pastagens (CAN+BRI, CAN+NAP e

Tabela 3 - Equações de regressão da produção de leite por animal (kg/vaca/ano) em cada nível de utilização de concentrado ( $\mathrm{kg} / \mathrm{vaca} / \mathrm{ano}$ e $\mathrm{R} \$ / \mathrm{vaca} / \mathrm{ano}$ ) e custos de alimentação (R\$/vaca/ano) para cada estratégia de alimentação e todas em conjunto (geral)

\begin{tabular}{lrc}
\hline $\begin{array}{l}\text { Estratégia de } \\
\text { alimentação }\end{array}$ & $\mathrm{R}^{2}$ \\
\hline \multicolumn{3}{c}{ Etilização de concentrados (kg/vaca/ano) } \\
SIM & $761,56815+3,06485 * \mathrm{~kg}$ conc./vaca & 97,05 \\
SIM+BRI & $1583,86058+2,07887 * \mathrm{~kg}$ conc./vaca & 99,05 \\
SIM+NAP & $1585,91476+2,03388 * \mathrm{~kg}$ conc./vaca & 99,06 \\
SIM+TIF & $1747,50633+1,98600 * \mathrm{~kg}$ conc./vaca & 99,14 \\
CAN+BRI & $1097,08071+1,99684 * \mathrm{~kg}$ conc./vaca & 99,57 \\
CAN+NAP & $1230,56242+1,90716 * \mathrm{~kg}$ conc./vaca & 99,06 \\
CAN+TIF & $1136,05727+1,95395 * \mathrm{~kg}$ conc./vaca & 99,45 \\
GERAL & $1683,74811+1,91065 * \mathrm{~kg}$ conc./vaca & 90,19
\end{tabular}

Gasto com concentrado (R \$/vaca/ano)

SIM $\quad-70,13240+6,34828 * \mathrm{R} \$$ conc./vac SIM+BRI $1028,35983+4,37790 * \mathrm{R} \$$ conc./vaca

$\mathrm{SIM}+\mathrm{NAP} \quad 1440,67886+4,24926 * \mathrm{R} \$$ conc. $/$ vaca

99,58

SIM+TIF $1670,87428+4,07325 * \mathrm{R} \$$ conc./vaca

99,54

99,47

CAN+BRI $\quad 807,01605+3,69686 * \mathrm{R} \$$ conc./vaca

99,96

CAN+NAP $1200,36141+3,56523 * \mathrm{R} \$$ conc./vaca 99,89

CAN+TIF $1364,21982+3,39466 * \mathrm{R} \$$ conc./vaca 99,74

GERAL $1655,90474+3,57144 * \mathrm{R} \$$ conc./vaca 88,30

Gasto com alimentação (R/vaca/ano)

$\begin{array}{lrll}\text { SIM } & -2906,78319+5,89821 * \mathrm{R} \$ \text { alim./vaca } & 99,88 \\ \text { SIM+BRI } & -575,10735+4,45023 * \mathrm{R} \$ \text { alim./vaca } & 99,45 \\ \text { SIM+NAP } & -222,62146+4,37746 * \mathrm{R} \$ \text { alim./vaca } & 99,27 \\ \text { SIM+TIF } & -84,09779+4,22435 * \mathrm{R} \$ \text { alim./vaca } & 98,61 \\ \text { CAN+BRI } & -100,08266+3,81875 * \mathrm{R} \$ \text { alim./vaca } & 99,72 \\ \text { CAN+NAP } & 234,75822+3,71445 * \mathrm{R} \$ \text { alim./vaca } & 99,61 \\ \text { CAN+TIF } & 363,04105+3,52489 * \mathrm{R} \$ \text { alim./vaca } & 98,94 \\ \text { GERAL } & -105,39510+4,01452 * \mathrm{R} \$ \text { alim./vaca } & 95,42\end{array}$

1 Todas as equações $P<0,01$.

$\mathrm{SIM}=$ silagem de milho como volumoso exclusivo; $\mathrm{SIM}+\mathrm{BRI}=$ silagem de milho durante a época seca e pastejo em capim-braquiária durante a época das águas; SIM+NAP = silagem de milho durante a época seca e pastejo de capim-elefante durante a época das águas; $\mathrm{SIM}+\mathrm{TIF}=$ silagem de milho durante a época seca e pastejo em capim-tifton 85 durante a época das águas; CAN+BRI = cana-de-açúcar durante a época seca e pastejo em capim-braquiária durante a época das águas; CAN+NAP = cana-de-açúcar durante a época seca e pastejo de capim-elefante durante a época das águas; CAN+TIF = cana-de-açúcar durante a época seca e pastejo em capim-tifton 85 durante a época das águas.
Tabela 4 - Produto físico marginal da produção de leite por animal (kg/vaca/ano) em cada nível de utilização de concentrado ( $\mathrm{kg} / \mathrm{vaca} / \mathrm{ano}^{1}$ e $\mathrm{R} \$ / \mathrm{vaca} / \mathrm{ano}^{3}$ ) e custo de alimentação (R\$/vaca/ano $\left.{ }^{4}\right)$ para cada estratégia de alimentação

\begin{tabular}{lrrrrrr}
\hline $\begin{array}{l}\text { Estratégia de } \\
\text { alimentação }\end{array}$ & PFma $^{1}$ & $\%^{2}$ & $\mathrm{PFma}^{3}$ & $\%$ & $\mathrm{PFma}^{4}$ & $\%$ \\
\hline SIM & 3,06 & 100,00 & 6,35 & 100,00 & 5,90 & 100,00 \\
SIM+BRI & 2,08 & 67,97 & 4,38 & 68,98 & 4,45 & 75,42 \\
SIM+NAP & 2,03 & 66,34 & 4,25 & 66,93 & 4,38 & 74,24 \\
SIM+TIF & 1,99 & 65,03 & 4,07 & 64,09 & 4,22 & 71,53 \\
CAN+BRI & 2,00 & 65,36 & 3,70 & 58,27 & 3,82 & 64,75 \\
CAN+NAP & 1,91 & 62,42 & 3,56 & 56,06 & 3,71 & 62,88 \\
CAN+TIF & 1,95 & 63,73 & 3,39 & 53,39 & 3,52 & 59,66 \\
\hline
\end{tabular}

$1 \mathrm{Em}$ relação à utilização de concentrados ( $\mathrm{kg} / \mathrm{vaca} / \mathrm{ano})$.

2 Porcentual em relação ao valor da estratégia SIM.

$3 \mathrm{Em}$ relação ao gasto com concentrados ( $\mathrm{R} \$$ conc./vaca/ano).

${ }^{4} \mathrm{Em}$ relação ao gasto com alimentação ( $\left.\mathrm{R} \$ / v a c a / a n o\right)$.

$\mathrm{SIM}=$ silagem de milho como volumoso exclusivo; $\mathrm{SIM}+\mathrm{BRI}=$ silagem de milho durante a época seca e pastejo em capim-braquiária durante a época das águas; SIM+NAP = silagem de milho durante a época seca e pastejo de capim-elefante durante a época das águas: $S I M+T I F=$ silagem de milho durante a época seca e pastejo em capim-tifton 85 durante a época das águas; $\mathrm{CAN+BRI} \mathrm{=} \mathrm{cana-de-açúcar} \mathrm{durante} \mathrm{a} \mathrm{época} \mathrm{seca} \mathrm{e} \mathrm{pastejo} \mathrm{em}$ capim-braquiária durante a época das águas: $\mathrm{CAN}+\mathrm{NAP}$ = cana-de-açúcar durante a época seca e pastejo de capim-elefante durante a época das águas; CAN+TIF = cana-de-açúcar durante a época seca e pastejo em capim-tifton 85 durante a época das águas.

CAN+TIF) apresentaram, na média, PFma correspondente a 66,4 e 63,8\%, quando avaliadas em $\mathrm{kg} / \mathrm{vaca} / \mathrm{ano}$, e 66,7 e $55,9 \%$, quando avaliadas em $\mathrm{R} \$ / \mathrm{vaca} / \mathrm{ano}$, em relação à estratégia SIM. Na média, o PFma, avaliado por kg de concentrado ou por real gasto com concentrado, respectivamente, das estratégias SIM+BRI, SIM+NAP e SIM+TIF foi de 2,03 e 4,23 por litro de leite produzido, enquanto, nas estratégias com utilização de cana-de-açúcar e pastagens (CAN+BRI, CAN+NAP eCAN+TIF), foi em média 1,95e 3,55 por litro de leite produzido.

A avaliação do PFma em relação ao custo de alimentação comprovou melhor eficiência na utilização dos alimentos da estratégia de alimentação SIM. Na média de todos os níveis de produção, essa estratégia produziu 5,9 litros de leite a cada real gasto com alimentação.

Comparando as estratégias de alimentação com utilização de silagem de milho (SIM+BRI, SIM+NAP e SIM+TIF) às estratégias à base de cana-de-açúcar (CAN+BRI, CAN+NAP e CAN+TIF), cujos valores foram, em média, de 4,35 e 3,68 litros de leite por real gasto com alimentação, respectivamente, esses valores representaram 73,73 e 62,43\% do valor obtido com a estratégia SIM.

Estes resultados demonstram que, se o objetivo é maximizar a receita e reduzir os custos de alimentação, considerando vacas de variados níveis de produção, a estratégia com utilização de silagem de milho como volumoso exclusivo apresentou os melhores resultados. Entretanto, a avaliação do PFma é realizada por animal e, como citado por Rennó 
et al. (2008), na avaliação de custos de alimentação por animal, pode ser utilizada se não houver limitações de área.

O resultado da avaliação do PFma para cada estratégia de alimentação resultou em conclusões semelhantes às obtidas por Nussio \& Nussio (2003) nas avaliações da receita menos os custos de alimentação por animal para as mesmas estratégias de alimentação.

\section{Conclusões}

A eficiência da utilização dos concentrados, quando avaliada em níveis crescentes de produção de leite por vaca, segue a lei dos retornos decrescentes, influenciando a eficiência bioeconômica quanto maior o nível de utilização de concentrados, característico de vacas de maior produção de leite. A maior eficiência biológica de vacas de maior produção de leite por lactação não resultou, necessariamente, em maior eficiência bioeconômica no cenário de preços de insumos e de leite analisados nesse estudo.

\section{Literatura Citada}

BATH, D.L.; SOSNIK, U. Formulation, delivery and inventory control of cost-effective rations. In: Van HORN, H.H.; WILCOX, C.J. (Eds.) Large dairy herd management. Savoy: American Dairy Science Association, 1992. p.709719.

BAUMAN, D.A.; McCUTCHEON, S.N.; STEINHOUR, W.D. et al. Sources of variation and prospects for improvement of productive efficiency in the dairy cow: a Review. Journal of Animal Science, v.60, p.583-595, 1985.

CONGLETON, W.R.; EVERETT, R.W. Application of the incomplete gamma function to predict cumulative milk production. Journal of Dairy Science, v.63, p.109-119, 1980.

FERREIRA, A. Eficiência de sistemas de produção de leite: uma aplicação da análise envoltória de dados na tomada de decisão. Viçosa, MG: Universidade Federal de Viçosa, 2002. 120p. Dissertação (Mestrado em Economia Rural) - Universidade Federal de Viçosa, 2002.

FOX, D.G.; TYLUTKI, T.P.; TEDESCHI, L.O. et al. The net carbohydrate and protein system for evaluating herd nutrition and nutrient excretion. CNCPS version 5.0. Ithaca: Cornell University, 2003. 292p. (Model documentation).

GIBSON, J.P. Efficiency and performance of genetically high and low milk-producing British Friesian and Jersey cattle. Animal Production, v.42, p.161-182, 1986

NATIONAL RESEARCH COUNCIL - NRC. Nutrient requirements of dairy cattle. 7.ed. Washington, D.C.: National Academic Press, 2001. 381p.

NUSSIO, L.G.; NUSSIO, C.M.B. Aspectos técnicos e econômicos que afetam a escolha da fonte de forragem suplementar. In SIMPÓSIO INTERNACIONAL DE PRODUÇÃO INTENSIVA DE LEITE - INTERLEITE, 6., 2003, Uberaba. Anais.. Uberaba: FMVZ-USP, 2003. p.123-135.

PEREIRA, J.C. Vacas leiteiras: aspectos práticos da alimentação. Viçosa, MG: Aprenda Fácil, 2000. 198p.

RENNÓ, F.P.; PEREIRA, J.C.; LEITE, C.A.M. et al. Avaliação bioeconômica de estratégias de alimentação em sistemas de produção de leite 1. Produção por animal e por área. Revista Brasileira de Zootecnia, v.35, n.4, p.****_****, 2008 .

STATISTICAL ANALYSIS SYSTEM - SAS. User'g Guide - Online Doc. v.8.0, Cary: 1999. (CR-ROM).

SCOTT CONSUlTORIA. A Nata do Leite, $\mathrm{n}^{\circ}$ 58-69. Bebedouro: 2003. p.8-12.

VANDEHAAR, M.J. Efficiency of nutrient use and relationship to profitability on dairy farms. Journal of Dairy Science, v.81, n.1, p.272-282, 1998. 